\title{
THE SPACE OF LEFT ORDERS OF A GROUP IS EITHER FINITE OR UNCOUNTABLE
}

\author{
PETER A. LINNELL
}

\begin{abstract}
Let $G$ be a group and let $\mathcal{O}_{G}$ denote the set of left orderings on $G$. Then $\mathcal{O}_{G}$ can be topologized in a natural way, and we shall study this topology to show that $\mathcal{O}_{G}$ can never be countably infinite.
\end{abstract}

\section{INTRODUCTION}

A group $G$ is left-ordered means that there is a total order $<$ on the group $G$ which is left invariant. Thus we have for all $g, x, y \in G$ with $x \neq y$ :

- Either $x<y$ or $y<x$, but never both.

- If $g<x<y$, then $g<y$.

- If $x<y$, then $g x<g y$.

In this situation, we shall write $(G,<)$ to mean the left-ordered group $G$ together with the given left order. Of course in general, there are many left orders on a leftordered group. However when $G$ is finite, $G$ has no left orders except in the case $G=1$, and then $G$ has exactly one left order.

We study the space $\mathcal{O}_{G}$ of all left orders on the left-ordered group $G$. Throughout this paper we let $\mathbb{N}=\{1,2, \ldots\}$, the positive integers, and for $a, g \in G$ we let $a^{g}=g a g^{-1}$. The topology on $\mathcal{O}_{G}$ is given by a base of open sets of the form

$$
U_{g_{1}, \ldots, g_{n}}:=\left\{<\in \mathcal{O}_{G} \mid g_{1}<\cdots<g_{n}\right\}
$$

where $g_{i} \in G$ and $n \in \mathbb{N}$. Another way to describe this topology is that it is given by the subbase $\left\{U_{1, g} \mid g \in G \backslash 1\right\}$. Of course to check that a map is continuous, we need only check that the inverse image of each element of the subbase is open, in particular if $f: X \rightarrow \mathcal{O}_{G}$ is a map from a topological space $X$, then $f$ is continuous if and only if $f^{-1}\left(U_{1, g}\right)$ is open in $X$ for all $g \in G \backslash 1$.

For $g_{1}, \ldots, g_{n} \in G$, set $V_{g_{1}, \ldots, g_{n}}=U_{1, g_{1}} \cap \cdots \cap U_{1, g_{n}}$. Then the sets $V_{g_{1}, \ldots, g_{n}}(0 \leq$ $n \in \mathbb{Z}$ ) form a base for the topology on $\mathcal{O}_{G}$. Sometimes we will write $V(G)_{g_{1}, \ldots, g_{n}}$ for $V_{g_{1}, \ldots, g_{n}}$ if it not clear with which group we are working with. An important property of $\mathcal{O}_{G}$ is that it is a totally disconnected compact Hausdorff space [11, Theorem 1.4]. In the case $G$ is countable, $\mathcal{O}_{G}$ is metrizable. Also there is a natural right $G$-action on $\mathcal{O}_{G}$ by homeomorphisms, where for $g \in G$ and $<\in \mathcal{O}_{G}$, we define

$$
x<_{g} y \Longleftrightarrow x^{g}<y^{g}
$$

for all $x, y \in G$. Thus $<_{g h}=\left(<_{g}\right)_{h}$ for all $g, h \in G$.

Date: Sat Sep 5 10:26:59 CEST 2009.

2000 Mathematics Subject Classification. Primary: 20F60; Secondary: 06F15.

Key words and phrases. left-ordered group, Cantor set. 
Recently Dave Witte Morris [8] gave a fabulous proof that a left-ordered amenable group is locally indicable. His method used the space $\mathcal{O}_{G}$ and some elementary ergodic theory. It would seem that $\mathcal{O}_{G}$ is worthy of further investigation.

If $(G,<)$ is a left-ordered group, then the positive cone of $G$ (relative to $<$ ) is $P:=\{g \in G \mid g>1\}$. Then $P$ satisfies the following:

Property 1.2. (a) If $g, h \in P$, then $g h \in P$.

(b) If $g \in P$, then $g^{-1} \notin P$.

(c) If $1 \neq g \in G$, then either $g$ or $g^{-1}$ is in $P$.

Conversely given a subset $P$ of $G$ satisfying (ㅁ) , (b) and (C) above, one can define a left order $<$ on $G$ by $h<g \Leftrightarrow h^{-1} g \in P$. Also one can use $P$ to give $G$ a right order, that is a total order $\prec$ which is right invariant, by defining $h \prec g$ if and only if $g h^{-1} \in P$. From this it is easy to see that a group is left-ordered if and only if it is right-ordered. For convenience, we only consider left-ordered groups. If $<_{g}=<$ for all $g \in G$, then $G$ is a bi-ordered group.

The purpose of this paper is to answer [7, Problem 16.51] in the negative. The problem asks "do there exist groups that can be right-ordered in infinitely countably many ways?" Considerable progress on this problem was made by A. V. Zenkov in 12, where he proved that the number of right orders on a locally indicable group is either finite or uncountable; see [5, Theorem 5.2.5]. We shall prove

Theorem 1.3. There is no group which can be left-ordered in a countably infinite number of ways.

A somewhat different proof of this theorem is given in [2, Theorem 3.1]. Very recently, another proof has been given in [1, Proposition 3.9]. Our proof will use the derived series (see \$2) of $\mathcal{O}_{G}$ to reduce to the case $G$ is locally indicable, and then to apply Zenkov's result above. The structure of groups with a nonzero finite number of left-orders is well-known; this is due to Tararin [5, Theorem 5.2.1]. Further information on this is given in [4].

I am very grateful to Adam S. Sikora for noticing a bad error in an earlier version of this paper [6]; this paper salvages the correct part of that paper. I would also like to thank Andrew Glass and Andrés Navas for encouraging me to write this paper.

\section{Preliminaries}

If $H$ is a subgroup of the group $G$, then any left order on $G$ restricts to a left order on $H$, so we have a well-defined restriction map $\rho_{G, H}: \mathcal{O}_{G} \rightarrow \mathcal{O}_{H}$, which is clearly continuous, because $\rho_{G, H}^{-1}\left(V(H)_{h}\right)=V(G)_{h}$ for all $h \in H$.

Let $X$ be an arbitrary Hausdorff topological space. Then the derived subset $X^{\prime}$ of $X$ is the subset obtained from $X$ by removing all its isolated points; equivalently $X^{\prime}$ is the set of limit points of $X$. Then $X^{\prime}$ is a closed subset of $X$. Of course, $X^{\prime}$ itself can still have isolated points, so for each ordinal $\alpha$, we define $X^{(\alpha)}$ by transfinite induction as follows.

- $X^{(0)}=X$.

- $X^{(\alpha+1)}=\left(X^{(\alpha)}\right)^{\prime}$.

- $X^{(\alpha)}=\bigcap_{\lambda<\alpha} X^{(\lambda)}$ if $\alpha$ is a limit ordinal.

It is clear that the subspaces $X^{(\alpha)}$ form a descending sequence of closed subspaces of $X$. For more details, see [10, $\S 8.5]$; in particular by [10, Theorem 8.5.2], there is an ordinal $\alpha$ such that $X^{(\alpha+1)}=X^{(\alpha)}$. We require the following easy lemma. 
Lemma 2.1. Let $X$ be a nonempty countable compact Hausdorff space. Then there exists an ordinal $\alpha$ such that $X^{(\alpha)}$ is finite and nonempty.

Proof. By [10, Proposition 8.5.7], let $\alpha$ be the least ordinal such that $X^{(\alpha)}=\emptyset$. Suppose $\alpha$ is a limit ordinal. Then $X^{(\alpha)}=\bigcap_{\lambda<\alpha} X^{(\lambda)}$. Since the $X^{(\lambda)}$ form a descending sequence of closed nonempty subsets of the compact space $X$, we see that $\bigcap_{\lambda<\alpha} X^{(\lambda)} \neq \emptyset$ and we have a contradiction. Therefore $\alpha$ must be a successor ordinal and we may write $\alpha=\beta+1$ for some ordinal $\beta$. Then $X^{(\beta)}$ is a nonempty compact Hausdorff space which consists only of isolated points, because $\left(X^{(\beta)}\right)^{\prime}=\emptyset$. Therefore $X^{(\beta)}$ is finite and nonempty.

\section{Proof of Theorem 1.3}

Proof of Theorem 1.3. Suppose by way of contradiction that $G$ is a left-ordered group such that $\mathcal{O}_{G}$ is countably infinite. We have a right $G$-action on $\mathcal{O}_{G}$ by homeomorphisms defined by $x<_{g} y$ if and only if $x^{g}<y^{g}$ (see (1.1)), and this will restrict to $G$-actions on $\mathcal{O}_{G}^{(\alpha)}$ for all ordinals $\alpha$. By Lemma 2.1, there is an ordinal $\beta$ such that $\mathcal{O}_{G}^{(\beta)}$ is finite and nonempty, and then there will be a normal subgroup $H$ of finite index in $G$ which fixes all the elements of $\mathcal{O}_{G}^{(\beta)}$. In particular, there is a left order $\prec$ on $G$ such that $\prec_{h}=\prec$ for all $h \in H$. Clearly $\rho_{G, H}(\prec)$ is a bi-order on $H$. Now by [9, Lemma 2.3], a bi-ordered group is of "Conrad type" 9, Section 2, p. 2570] (cf. [3. Lemma 6.6.2(1,3), p. 121]) and [9, Theorem 2.4] states that if $\rho_{G, H}(\prec)$ is of Conrad type and $H$ has finite index in $G$, then $\prec$ is also of Conrad type. Furthermore by [9, Theorem 4.1], a group is of Conrad type if and only if it is locally indicable. We conclude that $G$ is locally indicable, and the result now follows from [5, Theorem 5.2.5].

\section{REFERENCES}

[1] Adam Clay. Free lattice ordered groups and the topology on the space of left orderings of a group. Preprint, available via http://arxiv.org/abs/0909.0273

[2] Adam Clay, Andrés Navas, and Cristóbal Rivas. A new characterization of Conrad's property for group orderings, with applications. Preprint, available via http://arxiv.org/abs/0901.0880.

[3] A. M. W. Glass. Partially ordered groups, volume 7 of Series in Algebra. World Scientific Publishing Co. Inc., River Edge, NJ, 1999.

[4] A. Kirk. Classification of groups which admit a finite number of distinct right-orders. Comm. Algebra, 34(9):3357-3386, 2006.

[5] Valeriŭ M. Kopytov and Nikolaŭ Ya. Medvedev. Right-ordered groups. Siberian School of Algebra and Logic. Consultants Bureau, New York, 1996.

[6] Peter A. Linnell. The topology on the space of left orderings of a group. Preprint, available via http://arXiv/math.GR/0606232.

[7] V. D. Mazurov and E. I. Khukhro, editors. The Kourovka notebook. Rossiǔskaya Akademiya Nauk Sibirskoe Otdelenie, Institut Matematiki im. S. L. Soboleva, Novosibirsk, 16th edition, 2006. Unsolved problems in group theory.

[8] Dave Witte Morris. Amenable groups that act on the line. Algebr. Geom. Topol., 6:2509-2518, 2006.

[9] Akbar Rhemtulla and Dale Rolfsen. Local indicability in ordered groups: braids and elementary amenable groups. Proc. Amer. Math. Soc., 130(9):2569-2577 (electronic), 2002.

[10] Zbigniew Semadeni. Banach spaces of continuous functions. Vol. I. PWN-Polish Scientific Publishers, Warsaw, 1971. Monografie Matematyczne, Tom 55.

[11] Adam S. Sikora. Topology on the spaces of orderings of groups. Bull. London Math. Soc., $36(4): 519-526,2004$. 
[12] A. V. Zenkov. On groups with an infinite set of right orders. Sibirsk. Mat. Zh., 38(1):90-92, ii, 1997.

Department of Mathematics, Virginia Tech, Blacksburg, VA 24061-0123, USA

E-mail address: plinnell@math.vt.edu

URL: http://www.math.vt.edu/people/plinnell/ 